\section{Epigenetic Landscape of and Non-coding RNA Involvement in Somatic Cell Reprogramming}

\author{
Yanxin $\mathrm{Xu}^{\#}$, Dan Ye $\mathrm{e}^{\#}$, Xudong Guo\#, Jiuhong Kang ${ }^{* \#}$ and Jiajie \\ $\mathbf{X i}$
}

Clinical and Translational Research Center of Shanghai First Maternity and Infant Health Hospital, Shanghai Key Laboratory of Signaling and Disease Research, School of Life Science and Technology, Tongji University, Shanghai, China

"Equal Contribution to the Manuscript

\begin{abstract}
The use of Induced Pluripotent Stem (iPS) cells, especially those reprogrammed from individual patients, has great potential for regenerative medicine and drug discovery. The transition from the somatic state to the iPS state involves multiple epigenetic events, including histone modification, DNA methylation and non-coding RNA (ncRNA). However, the mechanisms underlying the epigenetic modifications regulating somatic cell reprogramming are largely unknown. Here, we present the recent discoveries regarding the involvement of epigenetic modifications in an IPs cell generation. Further understanding the role of epigenetic regulation in this reprogramming process would help to accelerate the clinical application of iPS cell technology.
\end{abstract}

In 2006, the discovery that somatic cells can be directly reprogrammed into an Embryonic Stem (ES) cell-like pluripotent state (iPS cells) via ectopic expression of the four transcription factors Oct4, Sox2, Klf4, and c-Myc, referred to as the Yamanaka factor, has reformed the conceptions of stem cell biology [1]. Although several limitations remain in the potential application of iPS cells [2], including low efficiency and genomic modification, this discovery is considered a powerful and encouraging tool for basic research and clinical applications. Because patient-specific pluripotent cells can be generated while avoiding ethical concerns, iPS technology is a promising strategy for the use of patient-derived iPS cells to study the pathogenesis of human diseases [3-6]. The iPS cells derived from patient-specific somatic cells typically contain the genetic

*Corresponding author: Jiuhong Kang, Clinical and Translational Research Center of Shanghai First Maternity and Infant Health Hospital, Shanghai Key Laboratory of Signaling and Disease Research, School of Life Science and Technology, Tongji University, Shanghai, China, Tel: 86-21-6598-8876; Fax: 86-21-6598-1041; E-mail: jhkang@tongji.edu.cn

Citation: Yanxin X, Dan Y, Xudong G, Jiuhong K, Jiajie X (2014) Epigenetic Landscape of and Non-coding RNA Involvement in Somatic Cell Reprogramming. J Stem Cell Res Dev Ther 1: 002.

Received: May 01, 2014; Accepted: July 08, 2015; Published: July 22, 2014 information that is responsible for the corresponding human disease [7-10]. Recently, a series of studies reported the establishment of various disease-specific human iPS cell lines, including cardiac disease [11-13], diabetes [14], blood disorders [15] and neurological disorders $[6,16,17]$. The specific somatic cells that are differentiated from these patients-specific iPS cells retain disease-related phenotypes, so these patient-specific iPS cell lines serve as in vitro cellular models to examine the molecular mechanisms of human diseases, screen new drugs and develop new therapies. iPS cells also hold great potential in the field of regenerative medicine $[18,19]$. Some trials using iPS cell-derived somatic cells have demonstrated some effectiveness in certain pathological animal models, including Parkinson's disease [20], hematopoietic diseases [21-24] and retinal dystrophy [25]. After performing genetic modifications to correct the defective genes via homologous recombination, iPS cells could also be applied as a therapy for some genetic mutation-related diseases, such as sickle cell anemia [23]. In addition to these diseases, iPS cell lines obtained from patients suffering from various diseases, including ALS, adenosine deaminase deficiency, Huntington disease, Gaucher disease, type 1 diabetes mellitus, muscular dystrophies, Down syndrome and Lesch-Nyhan syndrome [10], indicate the potential application of iPS cells to the treatment of these diseases. Given the potential of iPS cells for basic and clinical research, many investigators are highly interested in the molecular mechanisms underlying the transformation of differentiated cells into iPS cells.

Selected from a list of 24 candidate genes which were implicated in the biology of ES cells, Takahashi and Yamanaka identified four factors, Oct4, Sox2, c-Myc, and Klf4, which could directly induce the generation of iPS cells from mouse embryonic or adult fibroblasts. Recent studies have found that there are two transcriptional waves during somatic cell reprogramming, an initial wave driven by Klf4/c-Myc and a second wave driven by Oct4/Sox2/Klf4 [26]. Both waves contain epigenetic modification bursts, including histone modifications, and a biphasic pattern of microRNA (miRNA) and mRNA expression. However, DNA methylation gradually occurred during somatic cell reprogramming. During the first wave, the permissive cells gained proliferation ability and lost the identity of Mouse Embryonic Fibroblasts (MEFs), undergoing the Mesenchymal-to-Epithelial Transition (MET), the initial step of somatic cell reprogramming [27]. During the MET process, there are comparable changes in cell morphology and gene expression, including the up-regulation of epithelial genes and the down-regulation of mesenchymal genes, from the mesenchymal-like state to the epithelial-like state. The zinc finger transcription factor Klf4 was shown to maintain the self-renewal of ES cells in long-term cultures [28]. During iPS reprogramming progress, Klf4 was found to directly interact with Oct4 and Sox2. In addition, Klf4 acted as a cofactor with Oct4 to activate Nanog by binding to the Nanog promoter [29]. Some reports proposed that Klf4 triggers the MET process during iPS generation by regulating epithelial gene expression [30-32]. Klf4 was found to simultaneously repress somatic genes and activate pluripotency factors during the reprogramming process [26]. Myc is known to be required for early gene activation [33]. Previous research demonstrated that the transcription factor Myc was included in Yamanaka's reprogramming cocktail due to its pivotal role 
in ES cell self-renewal via its function as a downstream effector of the LIF/STAT3 pathway-mediated [34]. In addition, Myc was reported to interact with histone acetyl-transferase (HAT) complexes and the ATP-dependent chromatin remodeling complex SWI/SNF, indicating that Myc mediates chromatin remodeling. Although the global binding pattern of Myc was separable from the other three factors in ES cells and iPS cells, Myc contributed to the initial reprogramming events by inducing the transcriptional repression of fibroblast-specific genes $[33,35,36]$. Furthermore, Yamanaka et al showed that the Myc family member L-Myc [37], which displays little transformation activity, promotes iPS generation. Additionally, during the second wave, the pluripotency process was established via the exogenous expression of the pluripotency factors Oct4/Sox $2 / \mathrm{Klf} 4$, transforming the cells from an intermediate to a mature state.

Among these factors, the POU domain transcription factor Pou5f1 (also known as Oct4) was identified as a crucial gene in the core transcription regulatory pathway of ES cells [38-42]. Currently, a single factor, Oct 4 , is sufficient to reprogram the somatic cells into iPS cells $[30,43,44]$. A recent study showed that Oct 4 is highly involved in the molecular wave that leads to the activation of pluripotent genes to establish an ES cell-like state [26]. Because Oct4 is the most important reprogramming factor, ectopic expression of Oct 4 alone together with different combinations of small molecule inhibitors induces somatic cell reprogramming. Self-renewal and lineage specialization in the pre-implanted embryo primarily relies on intrinsic regulators, such as the transcription factor Sox $2[36,45]$. Recently, Sox 2 was found to bind to the promoter regions of the mir- $200 \mathrm{a} / \mathrm{b} / 429$ cluster and activate miR-200 family genes during iPS generation [46] Furthermore, mechanistic study of Yamanaka factor-mediated reprogramming revealed a transcriptional basis for the requirement of Sox2 during reprogramming [26,30].

The modulation of regulatory genes underlying iPS formation has been extensively studied and reviewed. The epigenetic state of cells is determined by their unique pattern of DNA methylation and histone modifications, which are responsible for the cell- and tissue-specific gene expression patterns. An emerging body of evidence suggested that during reprogramming, the mechanisms responsible for altering the epigenetic status of both DNA and histones also play important roles in iPS generation. Here, we provide a brief overview of iPS formation and the current state of knowledge regarding the roles of epigenetic modifications and ncRNA functions in this process.

Epigenetics was initially defined as "the study of mitotically and/or meiotically heritable changes in gene function that could not be explained by changes in DNA sequence" by Riggs et al. [47]. In 2008, at a Cold Spring Harbor Meeting, a consensus definition of a epigenetic trait was deemed as a "stably heritable phenotype resulting from changes in a chromosome without alterations in the DNA sequence" [48]. Currently, epigenetic research has converged on the study of modifications in DNA and histones and the influence of these modifications on gene expression $[49,50]$. These modifications include histone modification, chromatin remodeling, DNA methylation and ncRNA-mediated regulation. Epigenetic regulation affects nearly all biological processes, including somatic cell reprogramming. For example, valproic acid (VPA), a histone deacetylase (HDAC) inhibitor, improved MEF reprogramming efficiency in the presence of all four Yamanaka factors or the Yamanaka factors except for c-Myc [51]. In addition, VPA facilitates the reprogramming of human fibroblasts induced using Oct4 and Sox2 [52]. Vitamin C (Vc) enhances iPS generation from both mouse and human somatic cells by alleviating cell senescence and enabling chromatin configuration, as well as by modulating the H3K36me2 level at the activated epithelial gene locus [53-55]. Furthermore, different combinations of a miRNA cocktail successfully and effectively reprogram somatic cells into a pluripotent state [56-58]. Therefore, in this review, we focus on the recent advances in the epigenetic landscape of and the involvement of ncRNA in somatic cell reprogramming.

\section{Histone Modifications}

Histones are highly alkaline proteins expressed in eukaryotic cell nuclei that package and organize DNA into special units called nucleosomes, which are composed of an octamer containing two molecules of each of the four histones ( $\mathrm{H} 2 \mathrm{~A}, \mathrm{H} 2 \mathrm{~B}, \mathrm{H} 3$, and $\mathrm{H} 4)$ [59-61]. Because gene expression occurs on chromatin, the wrapping of the genomic DNA around histones is now known to be a critical characteristic of the regulation of gene expression. Post-translational modifications of histones alter their interaction with DNA and nuclear proteins, thereby affecting gene expression. Histones are primarily post-translationally modified on their N-terminal tails, but occasionally in their globular domains, by specific enzymes. Multiple dynamic modifications regulate gene transcription in a systematic and reproducible manner defined as the histone code. In an early study in 1964, Allfrey et al. found that histones could be acetylated and methylated and these modifications were found to affect RNA synthesis. To date, more than 130 sites of post-transcriptional covalent histone modifications, including propionylation, butyrylation, formylation, phosphorylation, ubiquitylation, sumoylation, citrullination, proline isomerization, ADP ribosylation, tyrosine hydroxylation, and lysine crotonylation, have been identified and characterized to be involved in biological processes. Among these modifications, histone methylation and acetylation are the most studied regarding somatic cell reprogramming [62].

\section{Methylation}

Histone methylation, one of the most studied histone modifications, is associated with both transcriptional activation and repression [63]. Trimethylated H3 lysines 9 and 27 (H3K9me3 and $\mathrm{H} 3 \mathrm{~K} 27 \mathrm{me} 3$ ) are repressive histone marks that are associated with gene repression in heterochromatin and euchromatin $[64,65]$. To establish the appropriate epigenetic code, the removal or addition of histone methylation marks, plays a key role in somatic cell reprogramming. Researchers identified H3K9 methylation as the primary epigenetic determinant modification for the intermediate state of reprogramming. Furthermore, they found that H3K9 demethylase promotes the transition from the pre-iPS to the completely reprogrammed iPS state [66]. A mechanistic study showed that H3K9 methyltransferase, Setdb1 and Suv39h1 are targeted by BMPs, arresting reprogramming at the pre-iPS stage, which is considered as a barrier to somatic cell reprogramming. Alternatively, Trithorax group (TrxG) and Polycomb group (PcG) protein complexes are key regulators of chromatin structures that are required for segmental identity in the developing embryo and contribute to the maintenance of the pluripotent state $[67,68]$. TrxG complexes catalyze histone H3 lysine4 trimethylation ( $\mathrm{H} 3 \mathrm{~K} 4 \mathrm{me} 3)$ at the promoters of protein-coding genes, miRNA loci and long non-coding RNA (lncRNA) loci, which are associated with the activation of transcription, whereas the PcG protein complex PRC2 catalyzes histone $\mathrm{H} 3$ lysine27 trimethylation (H3K27me3), which is associated with transcriptional repression [69-72]. Similar to H3K4me3, trimethylated H3 lysine36 (H3K36me3), 
which is also frequently located in transcriptionally active euchromatic regions, was initially detected predominantly at gene promoters and subsequently in the bodies of genes [73]. A previous report showed that Oct 4 transcriptionally activates Wdr5, a core member of the mammalian TrxG complex, to enhance its expression during somatic cell reprogramming [74]. Moreover, Wdr5 co-operates with Oct4 to establish a high $\mathrm{H} 3 \mathrm{~K} 4 \mathrm{me} 3$ level at the promoters of Oct4 itself and Nanog. Taken together, the Oct $4 /$ Wdr 5 feedback loop facilitates the establishment of pluripotency during the reprogramming process. Furthermore, this loop is involved in the regulation of ES cell self-renewal. Additionally, histone demethylases have been found to be directly involved in somatic cell reprogramming, as two well-known Vc-dependent H3K36 demethylases, Jhdm1a/Jhdmlb, play a pivotal role in the reprogramming process by counteracting p53/p21-induced cell senescence and by interacting with Oct 4 to activate the expression of the miRNA-302/367 cluster, which was previously demonstrated to be involved in the pluripotency process [53]. In addition, the miRNA-302/367 cluster alone was previously reported to induce somatic cells to obtain a pluripotent state. Another H3K36 demethylase, $\mathrm{Kdm} 2 \mathrm{~b}$, was also reported to promote somatic cell reprogramming. By up regulating the expression level of epithelial genes, $\mathrm{Kdm} 2 \mathrm{~b}$ promotes the MET, the critical step of early reprogramming, which, in turn, facilitates the activation of pluripotent transcription factors. Although a previous study demonstrated that $\mathrm{Kdm} 2 \mathrm{~b}$ could suppress cellular senescence, the rate of cell proliferation during the reprogramming process was not accelerated in the presence of $\mathrm{Kdm} 2 \mathrm{~b}$, indicating that $\mathrm{Kdm} 2 \mathrm{~b}$ promoted the somatic cell reprogramming in a proliferation-independent manner [75].

\section{Acetylation}

The lysine tails attached to the end of histones are positively charged. The acetylation of these tails neutralizes these chromatin ends, allowing for DNA access [76]. Thus, both histone acetylation and deacetylation are essential histone modifications that regulate transcriptional activity. It is known that the level of histone acetylation, which is regulated by the balance between Histone Acetylase (HAT) and Histone Deacetylase (HDAC) activity, generally correlates to the activation of gene transcription [77]. Recent studies have provided further evidence demonstrating the distinctly important roles of histone acetylation in iPS generation. Several groups reported that HDAC inhibitors rectify the aberrant silencing of loci and eliminate the epigenetic memory of somatic cells in established iPS cell lines. During the reprogramming process, treating the somatic cells with an HDAC inhibitor, such as VPA, TSA, SAHA, or butyrate, significantly improves the efficiency of iPS generation [51,52,78,79]. Melton's group found that the HDAC inhibitor VPA improves the reprogramming efficiency by more than 100 -fold in the presence of all four Yamanaka factors or the Yamanaka factors except for c-Myc based on the expression of the reporter Oct4-GFP [51]. After several months, they observed that VPA could enable the reprogramming of human fibroblasts induced using Oct4 and Sox2 [52]. Another HDAC inhibitor, butyrate, has been demonstrated to be associated with iPS generation, as well. Liang et al. showed that butyrate significantly facilitates somatic cell reprogramming and reduces the frequency of partially reprogrammed cells by improving the reprogramming fidelity, altering both the reprogramming dynamics and the ratio of iPS cell colonies to total colonies [78]. Furthermore, Mali et al. found that butyrate enhances histone $\mathrm{H} 3$ acetylation, promoting DNA demethylation and the expression of endogenous pluripotency-associated genes, which increases the efficiency of human iPS generation from fibroblasts [79]. The histone deacetylation-associated repressor complex Mbd3/NuRD was found to be highly involved in iPS generation. In 2013, Hanna's group reported that depleting Mbd3 expression increases the reprogramming efficiency to nearly $100 \%$ in both mouse and human fibroblasts [80].

Anokye-Danso et al. found that ectopic expression of the miR-302/367 cluster rapidly and efficiently reprograms mouse and human somatic cells into iPS cells without any requirement for the Yamanaka factors, although the suppression of HDAC2 was also required for this process [56]. This study showed that miRNAs cooperate with HDAC-mediated epigenetic signaling in a powerful manner, thereby playing an essential role in the reprogramming process. Furthermore, Zhuang et al. demonstrated that class IIa HDACs and myocyte enhancer factor-2 (MEF2) proteins constitute a cascade that regulates iPS generation. MEF2 blocks the reprogramming process by inducing TGF- $\beta$ cytokines, which are highly associated with the MET, and class IIa HDACs tend to suppress the activity of MEF2, promoting the MET and, in turn, enhancing the reprogramming efficiency [81].

\section{Chromatin Remodeling}

Chromatin is a highly organized structure consisting of DNA, histones, non-histone proteins and RNA in the eukaryotic genome. Chromatin remodeling, an enzyme-assisted nucleosome architecture reformation process, regulates the access of condensed genomic DNA to regulatory transcription machinery proteins. Recent studies found that the spatial organization of chromatin between the open and closed conformations at specific regions determines gene expression and cell fate. Two major classes of protein complexes are involved in this process: histones and ATP-dependent chromatin remodeling complexes [82]. Whereas the chromatin structure in pluripotent stem cells is open and highly dynamic, that in somatic cells is an inactive heterochromatin conformation, implicating that chromatin remodeling is involved in somatic cell reprogramming. In a previous study, researchers identified the SWI/SNF complex, a component of the BAF chromatin remodeling complex, promotes somatic cell reprogramming [83]. The activity of Brg1 and Baf155, two subunits of the SWI/SNF complex, accounts for the enhancement in the reprogramming efficiency. Brgl and Baf155 increase the number of active histone methylation marks at the promoters of pluripotency-specific genes by converting the chromatin from a passive inactive state to a euchromatin state. In addition, the active chromatin state induced by Brg1 and Baf155 enhances the binding of the reprogramming factor Oct 4 to downstream reprogramming gene promoters to establish a pluripotent state. Furthermore, in a recent study, long-range chromosomal interactions were found to occur prior to the activation of endogenous Oct 4 during the reprogramming process. Researchers showed that the reprogramming factor Klf4 interacts with the cohesin complex, which mediates DNA looping between enhancers and promoters, and recruits cohesin to the enhancer regions of the Oct4 locus. In turn, Oct4 and other pluripotent genes are reactivated by the formation of these intra-chromosomal loops. In addition, knockdown of the cohesion-complex gene SMC1 was suggested to abolish these intra-chromosomal interactions and this induction of pluripotency [84]. 


\section{DNA Methylation}

DNA methyltransferases (DNMTs) regulate DNA methylation by recognizing CpGs and catalyzing the transfer of a methyl group to the cytosine residues on DNA. It is well established that high CpG methylation in a promoter region correlates to the inhibition of the expression of the corresponding gene. DNA methylation has also been considered as a major epigenetic barrier to somatic cell reprogramming. Mikkelsen et al. suggested that a large portion of cells treated with the four Yamanaka factors are unable to overcome this major reprogramming barrier and are trapped in a partially reprogrammed state. Treatment of mouse fibroblasts with the DNMT inhibitor 5-Azacytidine (5-Aza) significantly increased the number of induced ES cell-like colonies and enhanced the reprogramming efficiency using all four Yamanaka factors or the Yamanaka factors except for c-Myc $[10,52,85]$. In the partially reprogrammed cells, the endogenous pluripotent genes were not completely reactivated because DNA hyper-methylation was retained at their promoters. 5-Aza treatment facilitates the transition from the partially reprogrammed state to the iPS state by reactivating these pluripotent genes, such as Oct4, Nanog, Utf1, and Dppa5, thereby promoting complete reprogramming. Treatment of partially reprogrammed cells with shRNAs reducing the level of Dnmt 1 expression also induced this stable transition [85]. The de novo DNA methyltransferases Dnmt3a and $3 \mathrm{~b}$ are directly recruited by G9a to the promoters of pluripotent genes, such as Oct4 and Rex1, during early embryogenesis, suggesting that these de novo DNMTs might also be involved in the re-activation of pluripotent genes [86].

Inappropriate or aberrant transcriptional silencing of imprinted genes via DNA methylation during the reprogramming process may shed the light on the developmental potential of iPS cells. Studies by Stadtfeld showed that the Dlk1-Dio3 region was predominantly aberrantly silenced by DNA methylation in Yamanaka factor-induced iPS cells and that the reactivation of this imprinted region might correlate to the degree of iPS pluripotency [87]. The addition of 5-Aza eliminated the improper DNA methylation marks from these imprinted genes and helped to establish iPS cells exhibiting complete differentiation potential, similar to ES cells [88]. The de novo DNMT Dnmt3a might be responsible for the hyper-methylation of the Dlk1-Dio3 locus and the aberrant silencing of this imprinted region during iPS generation, which was prevented by VPA treatment [89] or miR-29b transduction [90]. A recent study also showed that treatment with ascorbic acid impedes the binding of Dnmt3a to the promoter of the Dlk1-Dio3 locus, thus preventing the loss of expression of these imprinting genes [89].

RG108, another DNMT inhibitor [91], enhances the reprogramming efficiency by 30 -fold when applied in combination with an L-calcium channel agonist BayK8644 (BayK) and HMT inhibitor (BIX-01294) in the presence of only Oct4 and Klf4 [92]. These findings demonstrated that DNMT inhibition plays a pivotal role in iPS generation and that DNA methylation is a critical epigenetic obstacle to overcome during the reprogramming process.

Recent studies convincingly demonstrated that active DNA demethylation, which is independent of DNA replication, is essential for rapid and stable reprogramming. Cytidine deaminases (activation-induced cytidine deaminase; AID) and 5-methylcytosine DNA hydroxylases (Tets) are two major classes of molecules that are known to be responsible for active DNA demethylation.
Bhutani et al. developed a strategy to examine the initiation of reprogramming by fusing mouse ES cells to human skin cells to generate hybrids referred to as heterokaryons [93]. AID [94] was shown to be required for demethylation at the promoter regions of two well-known pluripotent genes: Oct4 and Nanog. A loss-of-function experiment using siRNA against AID showed that knockdown of AID remarkably repressed the demethylation and reactivation of expression of Nanog and Oct 4 in heterokaryons. These results suggested that demethylation of these two key pluripotent genes by AID is an essential step in reprogramming and demonstrated that the effect of AID on active DNA demethylation is crucial for the reprogramming process. In addition, it has been suggested that global genomic demethylation in Primordial Germ Cells (PGCs) [95] and in zebrafish embryos [96] might occur via an AID-mediated active demethylation mechanism. A recent study confirmed that AID does bind to methylated gene regions but not to unmethylated regions based on an AID ChIP assay and that AID cooperates with the Gadd 45 protein to demethylate DNA during DNA repair [96]. The deaminase activity of AID is critical for the initiation of reprogramming, and loss of AID significantly impairs the reprogramming efficiency by $80 \%$ [97]. In addition, AID was suggested to be involved in the regulation of epigenetic memory based on an assay of differentiated cells lacking AID during the reprogramming process from the somatic to iPS state. The results showed that AID-lacking cells maintained a hypermethylated genome, displayed low expression levels of pluripotent genes and failed to remain stabilized in the pluripotent state [98]. These findings emphasized the effect of AID on DNA demethylation and the crucial role of AID-mediated active DNA demethylation in reprogramming.

Several studies identified that Tet-mediated active DNA demethylation plays an important role in somatic cell reprogramming. Recent findings indicated that Tet1, which always binds to the transcriptional start sites of genes, might help to prevent aberrant DNA methylation at CpG-rich promoters [99], suggesting that DNA hydroxymethylation might play an critical role in genome-wide epigenetic remodeling during reprogramming. Gao's group also found that Tet 1 directly promotes Oct 4 demethylation and reactivation, facilitating iPS generation. Their finding implicated that Tet 1 could substitute for Oct 4 to trigger the initiation of molecular events involved in the reprogramming process and that both 5-methylcytosine and 5-hydroxymethylcytosine (5hmC) modifications are increased during reprogramming [100]. It is known that enrichment of $5 \mathrm{hmC}$ in gene regions during reprogramming is required for effective gene demethylation and reactivation. Although all Tet family members (Tet1, Tet2 and Tet3) catalyze the conversion of $5 \mathrm{mC}$ to $5 \mathrm{hmC}$ [101], only Tet 1 is involved in the maintenance of pluripotency and is required for the regulation of Nanog expression in ES cells, which negatively correlates to the level of DNA methylation at its promoter [102]. Tet1 and Nanog may also interact to promote the hydroxylation of Oct 4 and Esrrb. It has been suggested that Tet1 is up regulated during iPS reprogramming and participates in the active DNA demethylation of pluripotency genes.

Tet2 may also physically associate with Nanog to enhance the efficiency of iPS formation [103]. Doege showed that the Yamanaka factors activate the expression of Tet2, but not Tet1 or Tet3, and act together with Parp1, a poly (ADP-ribose) polymerase, to prevent further methylation of the Nanog and Esrrb promoters and to enhance the hydroxylation of these pluripotent genes [104], indicating that Tet2 might predominantly mediate the hydroxylation, demethylation, 
and reactivation of genes during the reprogramming process. Tet2 is also responsible for the rapid accumulation of 5-hmC at the loci of pluripotent genes, such as Oct4, during the reprogramming process in newly formed heterokaryons from the fusion of differentiated cells to pluripotent cells [105]. Consistently, Tet2-depleted mouse fibroblasts are unable to generate iPS cells in the presence of the Yamanaka factors because the MET is blocked by Tet2 deficiency. Reactivation of the key miRNAs (miR-200 family), depending on oxidative demethylation promoted by Tet and TDG sequentially, partially alleviates this inhibition of iPS generation [106].

Vc has been reported to interact with the catalytic domains of Tet1 and Tet 2 and to enhance their enzymatic activity. The addition of Vc was also reported to increase the genome-wide 5-hmC level $[107,108]$. Yin et al. showed that the global 5-mC levels in ES cells decreased by $40 \%$ in the presence of $\mathrm{Vc}$ for 3 days [108]. Thus, oxidative demethylation to promote pluripotency gene reactivation was suggested to be functionally required for the reprogramming of fibroblasts into iPS cells.

\section{miRNAs}

miRNAs are hairpin-derived small ncRNAs containing 22-24 nucleotides. miRNA-coding genes are transcribed into long primary miRNAs (pri-miRNAs) that are processed into precursors, defined as pre-miRNAs, by the DGCR8 complex and are then exported and processed into mature base-paired miRNAs by Drosha and Dicer [61]. miRNAs may act as post-transcriptional regulators to repress gene expression by binding to the 3'UTRs, the 5'UTRs and the coding sequences of target genes [109]. By post-transcriptionally modulating gene regulation, miRNAs are likely involved in most biological processes and are known to play important roles in somatic cell reprogramming.

Recently, several studies showed that the combination of a few miRNAs can successfully and effectively reprogram somatic cells into a pluripotent state $[56,58]$. Interestingly, miR-302 was the common factor in these three studies. miR-302 was initially reported to induce pluripotency independently of transcription factors, by the Wu's group in 2008. Direct transfection of mature miRNAs may reduce the risk of tumorigenesis and mutations without genomic integration. Wu et al. introduced a doxycycline-induced construct of miR-302a-d into normal human hair follicle cells via electroporation. Approximately 5-6 days after inducing miR-302 expression, more than $90 \%$ of the cells had been reprogrammed, displaying the expression of pluripotency markers, such as Oct4, Sox2, and Nanog [57]. Further investigation revealed that miR-302 enhances multiple aspects of reprogramming $[31,110]$. First, miR-302 and -372 have been found to regulate the MET, which is an early event of reprogramming that is primarily triggered by Klf4. Researchers found that miR-302 and -372 negatively regulate TGF- $\beta$ signaling to increase the rate of the MET by directly repressing the expression of TGF- $\beta$ receptor 2 and the TGF- $\beta$ superfamily ligands LEFTY1 and LEFTY 2 during human fibroblast reprogramming [31]. Furthermore, the induction of bone morphogenetic protein (BMP) signaling may also promote the MET and the reprogramming process, and miR-302 has been shown to play a role in the regulation of BMP signaling during reprogramming [110]. Lipchina et al. showed that miR-302 targets multiple inhibitors of BMP signaling (e.g., TOB2, DAZAP2, and SLAIN1) in hESCs. Inhibition of miR-302 and -367 using miRNA antagonists decreased the expression level of Inhibitor of DNA Binding 1 (ID1) and T-cell Leukemia Homeobox 2 (TLX2), both of which are downstream genes of BMP signaling [111]. Second, miR-302 and other miRNAs, including miR-291, -294, and -295, defined as ES Cell-specific Cell cycle regulating (ESCC) miRNAs, regulate the G1/S phase transition in ES cells [112]. Compared to somatic cells, ES cells exhibit a high rate of cell proliferation and a shorter G1 phase [113,114]. It was reported that ectopic expression of miR-302 in human fibroblasts accelerated the G1/S transition [115]. Moreover, Judson reported that transient transfection with ESCC miRNAs in the presence of three transcription factors (Oct4, Klf4, and Sox2) enhance the formation of mouse iPS cells [116]. Furthermore, miR-302 is thought to enhance the generation of iPS cells by targeting and repressing p21 and the retinoblastoma family member Retinoblastoma-like 2 (RBL2), inhibiting the cell proliferation rate [117]. miR-302 has also been reported to function in resetting the chromosome and enhancing reprogramming by targeting several chromatin-modifying enzymes, including AOF1, AOF2 (also known as KDM1B and KDM1A, respectively), and the methyl-CpG-binding proteins GATAD2B and MECP2 [57]. It is possible that the miR-302-367 cluster reprograms differentiated cells into iPS cells by targeting and down regulating epigenetic repressors, which bind to the promoters of pluripotent genes. $\mathrm{Hu}$ reported that miR-302s also promotes induced pluripotency by targeting NR2F2, which directly inhibits Oct4 expression [118]. It remains to be clarified which chromatin regulators that are targeted directly or indirectly by the miR-302-367 cluster are responsible for triggering and amplifying these epigenetic modifications.

In addition to the miR-302-367 cluster, many other miRNAs have been reported to be involved in the regulation of somatic cell reprogramming. The miR-205 and miR-200 families have also been shown to enhance reprogramming by facilitating the MET in both mouse and human cells during the early step of reprogramming and to initiate the MET in canine mesenchymal cells by directly targeting and repressing Zinc Finger E-box Binding Homeobox 1 (ZEB1) and ZEB2, which serve as the primary repressors of Ecadherin $[119,120]$. Further, the miR-141/200c and miR-200a/b/429 clusters are activated by exogenous Oct4 and Sox2, respectively, and BMP signaling strongly induces the expression of both the miR200 family and miR-205, thereby promoting the MET in MEFs [31,46,120]. In addition, it has been reported that miR-130b, $-301 \mathrm{~b}$, and -721 enhance reprogramming by reducing the expression of the transcription factor Meox2, which is also involved in the MET [121].

let-7 miRNAs antagonize the function of ESCC miRNAs. Melton demonstrated that knockdown of let-7 significantly improves reprogramming [122]. Moreover, miR-294 might enhance reprogramming by increasing the expression of LIN28, which has been shown to regulate the biogenesis of let-7 in ES cells [122,123]. The opposition of the effect of let-7 on reprogramming might partially explains the mechanism by which ESCC miRNAs (miR-291, -294, -295 and -302) promote the generation of iPS cells in the presence of either three or four Yamanaka factors [116].

Cellular senescence has been identified as a critical barrier to the generation of iPS cells. Ectopic expression of reprogramming factors also leads to the up regulation of p53 and p21 and the induction of the DNA damage response, both of which result in cell senescence. Several groups have focused on the functional links between miRNAs and the p53 pathway during reprogramming. Over-expressing miR-138 enhances OSK (Oct4, Sox2, and Klf4)-mediated reprogramming by directly targeting and repressing p 53 expression [124]. Suppressing miRNA-21 and $-29 \mathrm{a}$ also reduces the p53 
expression level by targeting CDC42 and p85a, respectively [125]. Importantly, the miRNA-34 family, which is activated by p53, prevents the generation of iPS cells by targeting Nanog and Sox2. The miRNA-34 family has emerged as a direct p53 target gene that mediates the induction of cell cycle arrest and senescence via p53 [126]. Furthermore, miRNA-199a-3p is up regulated by p53 and negatively regulates somatic cell reprogramming [127]. miR-199a-3p might function as another mediator of p53 and has recently been reported to target p21-activating kinase 4 (PAK4), thereby suppressing HCC growth [128]. In contrast, either over-expression of miR-199a-3p or deletion of PAK4 leads to an increase in the p21 expression level $[127,129,130]$

\section{lncRNAs}

The discovery of lncRNAs from the so-called transcriptional noise during the last decade has gradually aroused interest in the field of epigenetic regulation. Additional evidence has been shown demonstrating the remarkable function of lncRNAs in different biological processes, including somatic cell reprogramming [131-134].

Loewer et al. discovered ten Long Intergenic ncRNAs (lincRNAs) whose expression levels are robustly elevated in iPS cells compared to ES cells. Their results also revealed that these reprogramming-associated lincRNAs are directly regulated by specific key pluripotency transcriptional factors. In this study, they found that linc-RoR enhances iPS generation, providing the first evidence that lincRNAs function during somatic cell reprogramming [135]. Previous studies proposed a hypothesis which separated the process of iPS generation into several transcriptional factor-based waves, such as the MET, maturation and stabilization or stochastic and hierarchic, implying that the processes of cell reprogramming are separable. Moreover, the promoter regions of many ES cell-expressed lncRNAs have been demonstrated to be bound and regulated by key pluripotency-associated transcription factors, such as Oct4, Sox2, and Nanog [136] Because the expression levels of these lncRNAs might be high relative to those of these key factors, the function of lncRNAs during somatic cell reprogramming is likely to be hierarchic, as well, based on the stepwise activation of the corresponding upstream transcription factors.

lncRNA may interact with chromatin-modifying complexes and mediate the epigenetic modification of target genes, resulting in the attenuation of the expression of key transcription factors [137]. Increasing evidence has indicated physical interactions between lincRNA and chromatin-modifying complexes, such as PcG $[138,139]$ and TrxG [140,141], which are associated with histone methylation. Kahlili et al. found that $\sim 20 \%$ lincRNAs physically binds to chromatin-modifying complexes in various cell types [142]. Another well-understood lincRNA, HOTAIR, has been revealed to tether PRC2 and Lysine-Specific Demethylase 1 (LSD1) by acting as a molecular scaffold to mediate histone methylation or demethylation, thus maintaining the silence of HOX genes [137]. As for histone acetylation, the lncRNA JADE has been demonstrated to transcriptionally activate Jade1, a key component in the HBO1 histone acetylation complex, and induces H4 acetylation [143]. A recent study of the function of linc-RoR in ES cells suggested that another intriguing function of lincRNA is its activity as an endogenous miRNA sponge to competitively repress pluripotency-related miRNAs [144]. Later, several other studies showed that lncRNAs act as an endogenous sponge of miRNAs that participate in multiple biological processes to regulate cell proliferation and tissue development [145,146]. Another study showed that the lncRNA ecCEBPA competes with genomic DNA to bind to DNMT1 with a high affinity, thus preventing the methylation of its target gene CEBPA. Deep sequencing of transcripts associated with DNMT1 combined with genome-scale methylation and expression analysis revealed that the RNA-DNMT1 interaction might globally modulate genomic DNA methylation [147]. These recently discovered mechanisms of lincRNA in the regulation network of pluripotency might imply a potential role of lincRNA in somatic cell reprogramming.

\section{Perspectives}

Since Yamanaka's group first reported that the transcription factors Oct4, Sox2, Klf4, and c-Myc can convert differentiated cells to a pluripotent state, iPS cell reprogramming has been regarded as a useful cell model to investigate the molecular mechanisms of cellular pluripotency and differentiation, as well as a potential resource for cell replacement therapy. Increasing the efficiency of the reprogramming process and elucidating the mechanisms underlying the generation of iPS cells have become new hotspots of cell biology. The recent discoveries in iPS reprogramming, especially those using high-throughput technologies, such as RNA-seq and ChIP-seq, have allowed us to focus on epigenetic regulation. The reprogramming of differentiated cells into pluripotent cells is accompanied by dramatic changes in the epigenetic profiles of these cells. Histone modifications, such as H3K36me3, and DNA methylation are major epigenetic barriers to reprogramming. Application of a set of miRNAs alone or in combination with reprogramming factors, such as Oct4, Sox2, Klf4, c-Myc and LIN28, could break this epigenetic barrier and drive somatic cells to acquire pluripotency. lincRNAs mediate the crosstalk of the epigenetic landscape and perform multiple functions during iPS cell reprogramming. lincRNAs both bind to chromatin-modifying complexes to regulate the epigenetic modification of target genes and act as competitive endogenous sponges to repress target miRNAs. Dissecting these epigenetic modifications during the reprogramming process will provide a novel understanding of the determination of cell fate between pluripotency and differentiation, as well as lead to the development of new methods to generate iPS cells more efficiently.

\section{Acknowledgments}

This work was supported by grants obtained from the Ministry of Science and Technology (grant numbers 2011CB965100, 2010CB944900, 2011CBA01100, 2012CB966603, 2013CB967600, and 2013CB967401), the National Natural Science Foundation of China (grant numbers 91219305, 31210103905, 31371510, 31101061, 31171432, 81170499, 31201107, 31301208, and 81201599), the Science and Technology Commission of Shanghai Municipality (grant number 12ZR1450900), IRT1168 from the Ministry of Education, sponsored by Shanghai Rising-Star Program (14QA1403900), the Specialized Research Fund for the Doctoral Program of Higher Education (20110072110039), the "Chen Guang" project from the Shanghai Municipal Education Commission and the Shanghai Education Development Foundation (12CG19) and the Fundamental Research Funds for the Central Universities (2000219099). 
Citation: Yanxin X, Dan Y, Xudong G, Jiuhong K, Jiajie X (2014) Epigenetic Landscape of and Non-coding RNA Involvement in Somatic Cell Reprogramming. J Stem Cell Res Dev Ther 1: 002.

\section{References}

1. Takahashi K, Yamanaka S (2006) Induction of pluripotent stem cells from mouse embryonic and adult fibroblast cultures by defined factors. Cell 126 : 663-676.

2. Hu BY, Weick JP, Yu J, Ma LX, Zhang XQ, et al. (2010) Neural differentiation of human induced pluripotent stem cells follows developmental principles but with variable potency. Proc Natl Acad Sci U S A 107: 4335-4340.

3. Hargus G, Cooper O, Deleidi M, Levy A, Lee K, et al. (2010) Differentiated Parkinson patient-derived induced pluripotent stem cells grow in the adult rodent brain and reduce motor asymmetry in Parkinsonian rats. Proc Nat Acad Sci U S A 107: 15921-15926.

4. Soldner F, Hockemeyer D, Beard C, Gao Q, Bell GW, et al. (2009) Parkinson's disease patient-derived induced pluripotent stem cells free of viral reprogramming factors. Cell 136: 964-977.

5. Devine MJ, Ryten M, Vodicka P, Thomson AJ, Burdon T, et al. (2011) Parkinson's disease induced pluripotent stem cells with triplication of the $\alpha$-synuclein locus. Nat Commun 2: 440

6. Dimos JT, Rodolfa KT, Niakan KK, Weisenthal LM, Mitsumoto H, et al (2008) Induced pluripotent stem cells generated from patients with ALS can be differentiated into motor neurons. Science 321: 1218-1221.

7. Gunaseeli I, Doss MX, Antzelevitch C, Hescheler J, Sachinidis A (2010) Induced pluripotent stem cells as a model for accelerated patient- and disease-specific drug discovery. Curr Med Chem 17: 759-766.

8. Beyene R, Boockvar JA (2008) Disease-specific induced pluripotent stem cells. Neurosurgery 63: 12

9. Jang J, Yoo JE, Lee JA, Lee DR, Kim JY, et al. (2012) Disease-specific induced pluripotent stem cells: a platform for human disease modeling and drug discovery. Exp Mol Med 44: 202-213.

10. Park IH, Arora N, Huo H, Maherali N, Ahfeldt T, et al. (2008) Disease-specific induced pluripotent stem cells. Cell 134: 877-886.

11. Moretti A, Bellin M, Welling A, Jung CB, Lam JT, et al. (2010) Patient-specific induced pluripotent stem-cell models for long-QT syndrome. N Engl J Med 363: 1397-1409.

12. Itzhaki I, Maizels L, Huber I, Zwi-Dantsis L, Caspi O, et al. (2011) Modelling the long QT syndrome with induced pluripotent stem cells. Nature 471: 225 229.

13. Malan D, Friedrichs S, Fleischmann BK, Sasse P (2011) Cardiomyocytes obtained from induced pluripotent stem cells with long-QT syndrome 3 recapitulate typical disease-specific features in vitro. Circ Res 109: 841-847.

14. Maehr R, Chen S, Snitow M, Ludwig T, Yagasaki L, et al. (2009) Generation of pluripotent stem cells from patients with type 1 diabetes. Proc Natl Acad Sci U S A 106: 15768-15773.

15. Raya A, Rodríguez-Pizà I, Guenechea G, Vassena R, Navarro S, et al (2009) Disease-corrected haematopoietic progenitors from Fanconi anaemia induced pluripotent stem cells. Nature 460: 53-59.

16. Zhang N, An MC, Montoro D, Ellerby LM (2010) Characterization of Human Huntington's Disease Cell Model from Induced Pluripotent Stem Cells. PLoS Curr 2: RRN1193.

17. Lee G, Papapetrou EP, Kim H, Chambers SM, Tomishima MJ, et al. (2009) Modelling pathogenesis and treatment of familial dysautonomia using patient-specific iPSCs. Nature 461: 402-406.

18. Cooper $O$, Hallett $P$, Isacson $O$ (2012) Using stem cells and iPS cells to discover new treatments for Parkinson's disease. Parkinsonism Relat Disord 18 Suppl 1: S14-16.

19. Lindvall O, Barker RA, Brüstle O, Isacson O, Svendsen CN (2012) Clinica translation of stem cells in neurodegenerative disorders. Cell Stem Cell 10: 151-155.
20. Wernig M, Zhao JP, Pruszak J, Hedlund E, Fu D, et al. (2008) Neurons derived from reprogrammed fibroblasts functionally integrate into the fetal brain and improve symptoms of rats with Parkinson's disease. Proc Natl Acad Sci U S A 105: 5856-5861.

21. Xu D, Alipio Z, Fink LM, Adcock DM, Yang J, et al. (2009) Phenotypic correction of murine hemophilia A using an iPS cell-based therapy. Proc Natl Acad Sci U S A 106: 808-813.

22. Ye L, Chang JC, Lin C, Sun X, Yu J, et al. (2009) Induced pluripotent stem cells offer new approach to therapy in thalassemia and sickle cell anemia and option in prenatal diagnosis in genetic diseases. Proc Natl Acad Sci U S A 106: 9826-9830.

23. Hanna J, Wernig M, Markoulaki S, Sun CW, Meissner A, et al. (2007) Treatment of sickle cell anemia mouse model with iPS cells generated from autologous skin. Science 318: 1920-1923.

24. Wada H, Kojo S, Kusama C, Okamoto N, Sato Y, et al. (2011) Successful differentiation to T cells, but unsuccessful B-cell generation, from B-cell-derived induced pluripotent stem cells. Int Immunol 23: 65-74.

25. Carr AJ, Vugler AA, Hikita ST, Lawrence JM, Gias C, et al. (2009) Protective effects of human iPS-derived retinal pigment epithelium cell transplantation in the retinal dystrophic rat. PLoS One 4: e8152.

26. Polo JM, Anderssen E, Walsh RM, Schwarz BA, Nefzger CM, et al. (2012) A molecular roadmap of reprogramming somatic cells into iPS cells. Cell 151 1617-1632.

27. Li R, Liang J, Ni S, Zhou T, Qing X, et al. (2010) A mesenchymal-to-epithelial transition initiates and is required for the nuclear reprogramming of mouse fibroblasts. Cell Stem Cell 7: 51-63.

28. Li Y, McClintick J, Zhong L, Edenberg HJ, Yoder MC, et al. (2005) Murine embryonic stem cell differentiation is promoted by SOCS-3 and inhibited by the zinc finger transcription factor Klf4. Blood 105: 635-637.

29. Wei Z, Yang $Y$, Zhang $P$, Andrianakos R, Hasegawa $K$, et al. (2009) Klf4 interacts directly with Oct4 and Sox 2 to promote reprogramming. Stem Cells 27: 2969-2978.

30. Chen J, Liu J, Yang J, Chen Y, Chen J, et al. (2011) BMPs functionally replace KIf4 and support efficient reprogramming of mouse fibroblasts by Oct4 alone. Cell Res 21: 205-212.

31. Samavarchi-Tehrani P, Golipour A, David L, Sung HK, Beyer TA, et al. (2010) Functional genomics reveals a BMP-driven mesenchymal-to-epithelial transition in the initiation of somatic cell reprogramming. Cell Stem Cell 7: 64-77.

32. Yori JL, Johnson E, Zhou G, Jain MK, Keri RA (2010) Kruppel-like factor 4 inhibits epithelial-to-mesenchymal transition through regulation of E-cadherin gene expression. J Biol Chem 285: 16854-16863.

33. Sridharan R, Tchieu J, Mason MJ, Yachechko R, Kuoy E, et al. (2009) Role of the murine reprogramming factors in the induction of pluripotency. Cell 136: 364-377.

34. Cartwright P, McLean C, Sheppard A, Rivett D, Jones K, et al. (2005) LIF/ STAT3 controls ES cell self-renewal and pluripotency by a Myc-dependent mechanism. Development 132: 885-896.

35. Kim J, Woo AJ, Chu J, Snow JW, Fujiwara Y, et al. (2010) A Myc network accounts for similarities between embryonic stem and cancer cell transcription programs. Cell 143: 313-324.

36. Shi W, Wang H, Pan G, Geng Y, Guo Y, et al. (2006) Regulation of the pluripotency marker Rex-1 by Nanog and Sox2. J Biol Chem 281: 23319-23325.

37. Nakagawa M, Takizawa N, Narita M, Ichisaka T, Yamanaka S (2010) Promotion of direct reprogramming by transformation-deficient Myc. Proc Natl Acad Sci U S A 107: 14152-14157.

38. Nichols J, Zevnik B, Anastassiadis K, Niwa H, Klewe-Nebenius D, et al. (1998) Formation of pluripotent stem cells in the mammalian embryo depends on the POU transcription factor Oct4. Cell 95: 379-391. 
Citation: Yanxin X, Dan Y, Xudong G, Jiuhong K, Jiajie X (2014) Epigenetic Landscape of and Non-coding RNA Involvement in Somatic Cell Reprogramming. J Stem Cell Res Dev Ther 1: 002.

39. Niwa H, Miyazaki J, Smith AG (2000) Quantitative expression of Oct-3/4 defines differentiation, dedifferentiation or self-renewal of ES cells. Nat Genet 24: $372-376$.

40. Rosner MH, Vigano MA, Ozato K, Timmons PM, Poirier F, et al. (1990) A POU-domain transcription factor in early stem cells and germ cells of the mammalian embryo. Nature 345: 686-692.

41. Schöler HR, Dressler GR, Balling R, Rohdewohld H, Gruss P (1990) Oct4: a germline-specific transcription factor mapping to the mouse t-complex. EMBO J 9: 2185-2195.

42. Schöler HR, Ruppert S, Suzuki N, Chowdhury K, Gruss P (1990) New type of POU domain in germ line-specific protein Oct-4. Nature 344: 435-439.

43. Li Y, Zhang Q, Yin X, Yang W, Du Y, et al. (2011) Generation of iPSCs from mouse fibroblasts with a single gene, Oct4, and small molecules. Cell Res 21: 196-204.

44. Kim JB, Greber B, Araúzo-Bravo MJ, Meyer J, Park KI, et al. (2009) Direct reprogramming of human neural stem cells by OCT4. Nature 461: 649-643.

45. Avilion AA, Nicolis SK, Pevny LH, Perez L, Vivian N, et al. (2003) Multipotent cell lineages in early mouse development depend on SOX2 function. Genes Dev 17: 126-140.

46. Wang G, Guo X, Hong W, Liu Q, Wei T, et al. (2013) Critical regulation of miR-200/ZEB2 pathway in Oct4/Sox2-induced mesenchymal-to-epithelial transition and induced pluripotent stem cell generation. Proc Natl Acad Sci U S A 110: 2858-2863.

47. Tagoh H, Melnik S, Lefevre P, Chong S, Riggs AD, et al. (2004) Dynamic reorganization of chromatin structure and selective DNA demethylation prior to stable enhancer complex formation during differentiation of primary hematopoietic cells in vitro. Blood 103: 2950-2955.

48. Berger SL, Kouzarides T, Shiekhattar R, Shilatifard A (2009) An operational definition of epigenetics. Genes Dev 23: 781-783.

49. Bird A (2007) Perceptions of epigenetics. Nature 447: 396-398.

50. Ledford H (2008) Language: Disputed definitions. Nature 455: 1023-1028.

51. Huangfu D, Maehr R, Guo W, Eijkelenboom A, Snitow M, et al. (2008) Induction of pluripotent stem cells by defined factors is greatly improved by small-molecule compounds. Nat Biotechnol 26: 795-797.

52. Huangfu D, Osafune K, Maehr R, Guo W, Eijkelenboom A, et al. (2008) Induction of pluripotent stem cells from primary human fibroblasts with only Oct4 and Sox2. Nat Biotechnol 26: 1269-1275.

53. Wang T, Chen K, Zeng X, Yang J, Wu Y, et al. (2011) The histone demethylases $\mathrm{Jhdm} 1 \mathrm{a} / 1 \mathrm{~b}$ enhance somatic cell reprogramming in a vitamin-C-dependent manner. Cell Stem Cell 9: 575-587.

54. Esteban MA, Wang T, Qin B, Yang J, Qin D, et al. (2010) Vitamin C enhances the generation of mouse and human induced pluripotent stem cells. Cell Stem Cell 6: 71-79.

55. Chen J, Guo L, Zhang L, Wu H, Yang J, et al. (2013) Vitamin C modulates TET1 function during somatic cell reprogramming. Nat Genet 45: 1504-1509.

56. Anokye-Danso F, Trivedi CM, Juhr D, Gupta M, Cui Z, et al. (2011) Highly efficient miRNA-mediated reprogramming of mouse and human somatic cells to pluripotency. Cell Stem Cell 8: 376-388.

57. Lin SL, Chang DC, Lin CH, Ying SY, Leu D, et al. (2011) Regulation of somatic cell reprogramming through inducible mir-302 expression. Nucleic Acids Res 39: 1054-1065.

58. Miyoshi N, Ishii H, Nagano H, Haraguchi N, Dewi DL, et al. (2011) Reprogramming of mouse and human cells to pluripotency using mature microRNAs. Cell Stem Cell 8: 633-638.

59. Kouzarides T (2007) Chromatin modifications and their function. Cell 128: 693-705.

60. Berger SL (2007) The complex language of chromatin regulation during transcription. Nature 447: 407-412.
61. Gibney ER, Nolan CM (2010) Epigenetics and gene expression. Heredity (Edinb) 105: 4-13.

62. Tan M, Luo H, Lee S, Jin F, Yang JS, et al. (2011) Identification of 67 histone marks and histone lysine crotonylation as a new type of histone modification. Cell 146: 1016-1028.

63. Greer EL, Shi Y (2012) Histone methylation: a dynamic mark in health, disease and inheritance. Nat Rev Genet 13: 343-357.

64. Kim J, Kim H (2012) Recruitment and biological consequences of histone modification of H3K27me3 and H3K9me3. ILAR J 53: 232-239.

65. Asp P, Blum R, Vethantham V, Parisi F, Micsinai M, et al. (2011) Genome-wide remodeling of the epigenetic landscape during myogenic differentiation. Proc Natl Acad Sci U S A 108: E149-158.

66. Chen J, Liu H, Liu J, Qi J, Wei B, et al. (2013) H3K9 methylation is a barrier during somatic cell reprogramming into iPSCs. Nat Genet 45: 34-42.

67. Müller J, Kassis JA (2006) Polycomb response elements and targeting of Polycomb group proteins in Drosophila. Curr Opin Genet Dev 16: 476-484.

68. Ringrose L, Paro R (2007) Polycomb/Trithorax response elements and epigenetic memory of cell identity. Development 134: 223-232.

69. Smith ST, Petruk S, Sedkov Y, Cho E, Tillib S, et al. (2004) Modulation of heat shock gene expression by the TAC1 chromatin-modifying complex. Nat Cell Biol 6: 162-167.

70. Cao R, Wang L, Wang H, Xia L, Erdjument-Bromage H, et al. (2002) Role of histone $\mathrm{H} 3$ lysine 27 methylation in Polycomb-group silencing. Science 298: 1039-1043.

71. Czermin B, Melfi R, McCabe D, Seitz V, Imhof A, et al. (2002) Drosophila enhancer of Zeste/ESC complexes have a histone $\mathrm{H} 3$ methyltransferase activity that marks chromosomal Polycomb sites. Cell 111: 185-196.

72. Müller J, Hart CM, Francis NJ, Vargas ML, Sengupta A, et al. (2002) Histone methyltransferase activity of a Drosophila Polycomb group repressor complex. Cell 111: 197-208.

73. Wagner EJ, Carpenter PB (2012) Understanding the language of Lys36 methylation at histone H3. Nat Rev Mol Cell Biol 13: 115-126.

74. Ang YS, Tsai SY, Lee DF, Monk J, Su J, et al. (2011) Wdr5 mediates self-renewal and reprogramming via the embryonic stem cell core transcriptional network. Cell 145: 183-197.

75. Liang G, He J, Zhang Y (2012) Kdm2b promotes induced pluripotent stem cell generation by facilitating gene activation early in reprogramming. Nat Cell Biol 14: 457-466.

76. Grunstein M (1997) Histone acetylation in chromatin structure and transcription. Nature 389: 349-352.

77. Yang XJ, Seto E (2008) Lysine acetylation: codified crosstalk with other posttranslational modifications. Mol Cell 31: 449-461.

78. Liang G, Taranova O, Xia K, Zhang Y (2010) Butyrate promotes induced pluripotent stem cell generation. J Biol Chem 285: 25516-25521.

79. Mali P, Chou BK, Yen J, Ye Z, Zou J, et al. (2010) Butyrate greatly enhances derivation of human induced pluripotent stem cells by promoting epigenetic remodeling and the expression of pluripotency-associated genes. Stem Cells 28: $713-720$.

80. Rais Y, Zviran A, Geula S, Gafni O, Chomsky E, et al. (2013) Deterministic direct reprogramming of somatic cells to pluripotency. Nature 502: 65-70.

81. Zhuang Q, Qing X, Ying Y, Wu H, Benda C, et al. (2013) Class Ila histone deacetylases and myocyte enhancer factor 2 proteins regulate the mesenchymal-to-epithelial transition of somatic cell reprogramming. J Biol Chem 288: 12022-12031.

82. Teif VB, Rippe K (2009) Predicting nucleosome positions on the DNA: combining intrinsic sequence preferences and remodeler activities. Nucleic Acids Res 37: 5641-5655. 
Citation: Yanxin X, Dan Y, Xudong G, Jiuhong K, Jiajie X (2014) Epigenetic Landscape of and Non-coding RNA Involvement in Somatic Cell Reprogramming. J Stem Cell Res Dev Ther 1: 002.

83. Singhal N, Graumann J, Wu G, Araúzo-Bravo MJ, Han DW, et al. (2010) Chromatin-Remodeling Components of the BAF Complex Facilitate Reprogramming. Cell 141: 943-955.

84. Wei Z, Gao F, Kim S, Yang H, Lyu J, et al. (2013) Klf4 organizes long-range chromosomal interactions with the oct4 locus in reprogramming and pluripotency. Cell Stem Cell 13: 36-47.

85. Mikkelsen TS, Hanna J, Zhang X, Ku M, Wernig M, et al. (2008) Dissecting direct reprogramming through integrative genomic analysis. Nature 454: 4955.

86. Feldman N, Gerson A, Fang J, Li E, Zhang Y, et al. (2006) G9a-mediated irreversible epigenetic inactivation of Oct-3/4 during early embryogenesis. Nat Cell Biol 8: 188-194.

87. Stadtfeld M, Apostolou E, Akutsu H, Fukuda A, Follett $P$, et al. (2010) Aberrant silencing of imprinted genes on chromosome 12qF1 in mouse induced pluripotent stem cells. Nature 465: 175-181.

88. Bao S, Tang F, Li X, Hayashi K, Gillich A, et al. (2009) Epigenetic reversion of post-implantation epiblast to pluripotent embryonic stem cells. Nature 461 : 1292-1295.

89. Stadtfeld M, Apostolou E, Ferrari F, Choi J, Walsh RM, et al. (2012) Ascorbic acid prevents loss of Dlk1-Dio3 imprinting and facilitates generation of all-iPS cell mice from terminally differentiated B cells. Nat Genet 44: 398-405, S1-2.

90. Guo X, Liu Q, Wang G, Zhu S, Gao L, et al. (2013) microRNA-29b is a novel mediator of Sox2 function in the regulation of somatic cell reprogramming. Cell Res 23: 142-156.

91. Brueckner B, Garcia Boy R, Siedlecki P, Musch T, Kliem HC, et al. (2005) Epigenetic reactivation of tumor suppressor genes by a novel small-molecule inhibitor of human DNA methyltransferases. Cancer Res 65: 6305-6311.

92. Shi Y, Desponts C, Do JT, Hahm HS, Schöler HR, et al. (2008) Induction of pluripotent stem cells from mouse embryonic fibroblasts by Oct 4 and Klf4 with small-molecule compounds. Cell Stem Cell 3: 568-574.

93. Bhutani N, Brady JJ, Damian M, Sacco A, Corbel SY, et al. (2010) Reprogramming towards pluripotency requires AID-dependent DNA demethylation. Nature 463: 1042-1047.

94. Morgan HD, Dean W, Coker HA, Reik W, Petersen-Mahrt SK (2004) Activation-induced cytidine deaminase deaminates 5-methylcytosine in DNA and is expressed in pluripotent tissues: implications for epigenetic reprogramming. J Biol Chem 279: 52353-52360.

95. Popp C, Dean W, Feng S, Cokus SJ, Andrews S, et al. (2010) Genome-wide erasure of DNA methylation in mouse primordial germ cells is affected by AID deficiency. Nature 463: 1101-1105

96. Rai K, Huggins IJ, James SR, Karpf AR, Jones DA, et al. (2008) DNA demethylation in zebrafish involves the coupling of a deaminase, a glycosylase, and gadd45. Cell 135: 1201-1212.

97. Bhutani N, Decker MN, Brady JJ, Bussat RT, Burns DM, et al. (2013) A critical role for AID in the initiation of reprogramming to induced pluripotent stem cells. FASEB J 27: 1107-1113.

98. Kumar R, DiMenna L, Schrode N, Liu TC, Franck P, et al. (2013) AID stabilizes stem-cell phenotype by removing epigenetic memory of pluripotency genes. Nature 500: 89-92.

99. Williams K, Christensen J, Pedersen MT, Johansen JV, Cloos PA, et al (2011) TET1 and hydroxymethylcytosine in transcription and DNA methylation fidelity. Nature 473: 343-348.

100.Gao Y, Chen J, Li K, Wu T, Huang B, et al. (2013) Replacement of Oct4 by Tet1 during iPSC induction reveals an important role of DNA methylation and hydroxymethylation in reprogramming. Cell Stem Cell 12: 453-469.

101. Tahiliani M, Koh KP, Shen Y, Pastor WA, Bandukwala H, et al. (2009) Conversion of 5-methylcytosine to 5-hydroxymethylcytosine in mammalian DNA by MLL partner TET1. Science 324: 930-935.
102. Ito S, D'Alessio AC, Taranova OV, Hong K, Sowers LC, et al. (2010) Role of Tet proteins in $5 \mathrm{mC}$ to $5 \mathrm{hmC}$ conversion, ES-cell self-renewal and inner cell mass specification. Nature 466: 1129-1133.

103. Costa Y, Ding J, Theunissen TW, Faiola F, Hore TA, et al. (2013) NANOG-dependent function of TET1 and TET2 in establishment of pluripotency. Nature 495: 370-374.

104.Doege CA, Inoue K, Yamashita T, Rhee DB, Travis S, et al. (2012) Early-stage epigenetic modification during somatic cell reprogramming by Parp1 and Tet2. Nature 488: 652-655.

105.Piccolo FM, Bagci H, Brown KE, Landeira D, Soza-Ried J, et al. (2013) Different roles for Tet1 and Tet2 proteins in reprogramming-mediated erasure of imprints induced by EGC fusion. Mol Cell 49: 1023-1033.

106. Hu X, Zhang L, Mao SQ, Li Z, Chen J, et al. (2014) Tet and TDG mediate DNA demethylation essential for mesenchymal-to-epithelial transition in somatic cell reprogramming. Cell Stem Cell 14: 512-522.

107. Blaschke K, Ebata KT, Karimi MM, Zepeda-Martínez JA, Goyal P, et al. (2013) Vitamin C induces Tet-dependent DNA demethylation and a blastocyst-like state in ES cells. Nature 500: 222-226.

108. Yin R, Mao SQ, Zhao B, Chong Z, Yang Y, et al. (2013) Ascorbic acid enhances Tet-mediated 5-methylcytosine oxidation and promotes DNA demethylation in mammals. J Am Chem Soc 135: 10396-10403.

109. Hobert O (2008) Gene regulation by transcription factors and microRNAs. Science 319: 1785-1786.

110.Subramanyam D, Lamouille S, Judson RL, Liu JY, Bucay N, et al. (2011) Multiple targets of miR-302 and miR-372 promote reprogramming of human fibroblasts to induced pluripotent stem cells. Nat Biotechnol 29: 443-448.

111.Lipchina I, Elkabetz Y, Hafner M, Sheridan R, Mihailovic A, et al. (2011) Genome-wide identification of microRNA targets in human ES cells reveals a role for miR-302 in modulating BMP response. Genes Dev 25: 2173-2186.

112.Wang Y, Baskerville S, Shenoy A, Babiarz JE, Baehner L, et al. (2008) Embryonic stem cell-specific microRNAs regulate the G1-S transition and promote rapid proliferation. Nat Genet 40: 1478-1483.

113. White J, Dalton S (2005) Cell cycle control of embryonic stem cells. Stem Cell Rev 1: 131-138.

114.Burdon T, Smith A, Savatier P (2002) Signalling, cell cycle and pluripotency in embryonic stem cells. Trends Cell Biol 12: 432-438.

115. Card DA, Hebbar PB, Li L, Trotter KW, Komatsu Y, et al. (2008) Oct4/ Sox2-regulated miR-302 targets cyclin D1 in human embryonic stem cells. Mol Cell Biol 28: 6426-6438.

116.Judson RL, Babiarz JE, Venere M, Blelloch R (2009) Embryonic stem cell-specific microRNAs promote induced pluripotency. Nat Biotechnol 27: 459-461.

117.Litovchick L, Sadasivam S, Florens L, Zhu X, Swanson SK, et al. (2007) Evolutionarily conserved multisubunit RBL2/p130 and E2F4 protein complex represses human cell cycle-dependent genes in quiescence. Mol Cell 26: 539-551.

118. Rosa A, Brivanlou AH (2011) A regulatory circuitry comprised of miR-302 and the transcription factors OCT4 and NR2F2 regulates human embryonic stem cell differentiation. EMBO J 30: 237-248.

119.Park SM, Gaur AB, Lengyel E, Peter ME (2008) The miR-200 family determines the epithelial phenotype of cancer cells by targeting the E-cadherin repressors ZEB1 and ZEB2. Genes Dev 22: 894-907.

120.Gregory PA, Bert AG, Paterson EL, Barry SC, Tsykin A, et al. (2008) The miR-200 family and miR-205 regulate epithelial to mesenchymal transition by targeting ZEB1 and SIP1. Nat Cell Biol 10: 593-601.

121.Pfaff N, Fiedler J, Holzmann A, Schambach A, Moritz T, et al. (2011) miRNA screening reveals a new miRNA family stimulating iPS cell generation via regulation of Meox2. EMBO Rep 12: 1153-1159. 
122. Melton C, Judson RL, Blelloch R (2010) Opposing microRNA families regulate self-renewal in mouse embryonic stem cells. Nature 463: 621-626.

123. Viswanathan C (2008) Selective blockade of microRNA processing by Lin28. Science 320: 97-100.

124. Ye D, Wang G, Liu Y, Huang W, Wu M, et al. (2012) MiR-138 promotes induced pluripotent stem cell generation through the regulation of the p53 signaling. Stem Cells 30: 1645-1654.

125. Yang CS, Li Z, Rana TM (2011) microRNAs modulate iPS cell generation. RNA 17: 1451-1460.

126. Choi YJ, Lin CP, Ho JJ, He X, Okada N, et al. (2011) miR-34 miRNAs provide a barrier for somatic cell reprogramming. Nat Cell Biol 13: 1353-1360.

127.Wang J, He Q, Han C, Gu H, Jin L, et al. (2012) p53-facilitated miR-199a-3p regulates somatic cell reprogramming. Stem Cells 30: 1405-1413.

128. Hou J, Lin L, Zhou W, Wang Z, Ding G, et al. (2011) Identification of miRNomes in human liver and hepatocellular carcinoma reveals miR-199a/ b-3p as therapeutic target for hepatocellular carcinoma. Cancer Cell 19: 232 243.

129. Nekrasova T, Minden A (2011) PAK4 is required for regulation of the cell-cycle regulatory protein $\mathrm{p} 21$, and for control of cell-cycle progression. $\mathrm{J}$ Cell Biochem 112: 1795-1806.

130. Nekrasova T, Minden A (2012) Role for p21-activated kinase PAK4 in development of the mammalian heart. Transgenic Res 21: 797-811.

131. Lee JT (2012) Epigenetic regulation by long noncoding RNAs. Science 338 1435-1439.

132. Mercer TR, Mattick JS (2013) Structure and function of long noncoding RNAs in epigenetic regulation. Nat Struct Mol Biol 20: 300-307.

133.Rinn JL, Chang HY (2012) Genome regulation by long noncoding RNAs. Annu Rev Biochem 81: 145-166.

134. Guttman M, Donaghey J, Carey BW, Garber M, Grenier JK, et al. (2011) lincRNAs act in the circuitry controlling pluripotency and differentiation. Nature 477: $295-300$

135. Loewer S, Cabili MN, Guttman M, Loh YH, Thomas K, et al. (2010) Large intergenic non-coding RNA-RoR modulates reprogramming of human induced pluripotent stem cells. Nat Genet 42: 1113-1117.
136. Sheik Mohamed J, Gaughwin PM, Lim B, Robson P, Lipovich L (2010) Conserved long noncoding RNAs transcriptionally regulated by Oct4 and Nanog modulate pluripotency in mouse embryonic stem cells. RNA 16: 324-337.

137. Tsai MC, Manor O, Wan Y, Mosammaparast N, Wang JK, et al. (2010) Long noncoding RNA as modular scaffold of histone modification complexes. Science 329: 689-693.

138. Klattenhoff CA, Scheuermann JC, Surface LE, Bradley RK, Fields PA, et al. (2013) Braveheart, a long noncoding RNA required for cardiovascular lineage commitment. Cell 152: 570-583.

139. Kaneko S, Bonasio R, Saldaña-Meyer R, Yoshida T, Son J, et al. (2014) Interactions between JARID2 and noncoding RNAs regulate PRC2 recruitment to chromatin. Mol Cell 53: 290-300.

140. Grote P, Wittler L, Hendrix D, Koch F, Währisch S, et al. (2013) The tissue-specific IncRNA Fendrr is an essential regulator of heart and body wall development in the mouse. Dev Cell 24: 206-214.

141. Cabianca DS, Casa V, Bodega B, Xynos A, Ginelli E, et al. (2012) A long ncRNA links copy number variation to a polycomb/trithorax epigenetic switch in FSHD muscular dystrophy. Cell 149: 819-831.

142. Khalil AM, Guttman M, Huarte M, Garber M, Raj A, et al. (2009) Many human large intergenic noncoding RNAs associate with chromatin-modifying complexes and affect gene expression. Proc Natl Acad Sci U S A 106: 1166711672.

143. Wan G, Hu X, Liu Y, Han C, Sood AK, et al. (2013) A novel non-coding RNA IncRNA-JADE connects DNA damage signalling to histone $\mathrm{H} 4$ acetylation. EMBO J 32: 2833-2847.

144.Wang Y, Xu Z, Jiang J, Xu C, Kang J, et al. (2013) Endogenous miRNA sponge lincRNA-RoR regulates Oct4, Nanog, and Sox2 in human embryonic stem cell self-renewal. Dev Cell 25: 69-80.

145. Cesana M, Cacchiarelli D, Legnini I, Santini T, Sthandier O, et al. (2011) A long noncoding RNA controls muscle differentiation by functioning as a competing endogenous RNA. Cell 147: 358-369.

146. Kallen AN, Zhou XB, Xu J, Qiao C, Ma J, et al. (2013) The imprinted H19 IncRNA antagonizes let-7 microRNAs. Mol Cell 52: 101-112.

147.Di Ruscio A, Ebralidze AK, Benoukraf T, Amabile G, Goff LA, et al. (2013) DNMT1-interacting RNAs block gene-specific DNA methylation. Nature 503: 371-376. 